\title{
A statistical-based approach for fault detection and diagnosis in a photovoltaic system
}

\author{
Elyes Garoudja, Fouzi Harrou, Member, IEEE, Ying Sun, Kamel Kara, Aissa Chouder, Santiago \\ Silvestre
}

\begin{abstract}
This paper reports a development of a statistical approach for fault detection and diagnosis in a PV system. Specifically, the overarching goal of this work is to early detect and identify faults on the DC side of a PV system (e.g., shortcircuit faults; open-circuit faults; and partial shading faults). Towards this end, we apply exponentially-weighted moving average (EWMA) control chart on the residuals obtained from the one-diode model. Such a choice is motivated by the greater sensitivity of EWMA chart to incipient faults and its lowcomputational cost making it easy to implement in real time. Practical data from a $3.2 \mathrm{KWp}$ photovoltaic plant located within an Algerian research center is used to validate the proposed approach. Results show clearly the efficiency of the developed method in monitoring PV system status.
\end{abstract}

\section{INTRODUCTION}

Energy based on traditional sources (e.g., fuel oil, gasoline and coal) has some negative impacts on the human general health as well as the ambient environment[1]. Also, to meet the requirements of the COP 21 Paris agreement on climate change, many countries in the world are trying to reduce their dependability on some conventional fossil sources. Towards this end, renewable sources of energy such as solar and wind [2] are very promising alternative resources to replace an important proportion of the traditional forms of energy. Photovoltaic (PV) system, as solar based energy source, is one of the most promising clean energy this last time.

During its functioning, PV system, and more particularly its DC side (PV array) can be subjected to several types of faults and anomalies (open circuits faults, short circuits faults, hotspot faults, total and partial shading faults, ...,etc.), which reduce both its energetic yield and its efficiency [3]. The central problem with DC side faults is that they are

Elyes Garoudja and Kamel Kara are with the Electronic Department, Blida 1 University Blida, Algeria, (g_lyes@ hotmail.fr, k.kara68@gmail.com). Fouzi Harrou and Ying Sun are with King Abdullah University of Science and Technology (KAUST), Computer, Electrical and Mathematical Sciences and Engineering Division,Thuwal23955900, (fouzi.harrou, ying.sun @kaust.edu.sa).

Aissa Chouder is with the Electrical Engineering Department University of m’sila Ichbilia Street, Algeria (aissachouder@gmail.com).

Santiago Silvestre is with the Electronic Engineering Departmen Univesitat Politécnica de Catalunya Barcelona, Spain (Santiago.silvestre@upc.ed). hardly discovered by the user till a complete standstill of the system [4] or even possible terrible consequences in some situations $[5,6]$.

Up to now, methods using to detect and diagnose faults in PV systems are ranked into three categories [4]: ProcessHistory Based methods, Signal-Processing Based methods, and finally Quantitative-Model Based methods.

The first category denotes Process-History Based methods. It is composed essentially of two main approaches: Statistical [7] and Machine Learning [8-10] approaches. The statistical one has been appeared in applying both descriptive and inferential statistics to detect wiring fault occurrence in PV systems [7]. In addition to the statistical approaches, machine learning ones have gained very remarkable attention in fault detection and diagnosis area. For instance, the application of supervised learning methods, such as Artificial Neural Network (ANN), applied in [8] to evaluate PV system performances under partial shading condition. ANN has been suggested also to detect short circuit occurrence in PV array [9]. Others machine learning based methods have been suggested in the literature such as [10] in which authors proposed the application of Bayesian neural network (BNN) and polynomial regression to predict the soiling fault effect on large scale PV systems.

However, the main drawback in using this category appears in the requirement of a relevant dataset to describe healthy and faulty operations, and which is not always the case.

Signal-Processing Based methods denotes the second category of fault detection and diagnosis methods, the main idea of these methods is to apply signal processing's advanced technics to diagnose the PV system faults. Several signal processing based methods have been suggested in this context, such as Time Domain Reflectometry (TDR) proposed in [11], and which has been applied at the aim of detecting the faults occurrences, localizing the faults positions, and finally diagnosing the exact faults natures (short circuit fault, open circuit faults or reverse polarity of the module fault).

Despite the fact that TDR can detect, localize, and diagnose faults in PV systems, it suffered of two essential drawbacks and which can be summed up as: the system should be turned off to be capable of applying the TDR method which affect the system productivity, and the need of a very sophisticated tools to introduce the input signal and analyze the reflected one [4] hence the cost issue. 
When assessing the signal processing based methods' strategy, fault detection and diagnosis technics' high cost has been remarked as its main issue.

To finish with, Quantitative-Model Based methods is the last faults detection and diagnosis category. In fact, several research works have been suggested in this regard, such as work of [12] in which authors propose a method to detect faults in PV system based on the evaluation of the PV system Performances, named Performance Comparison method and which compares the real and the simulated (expected) performances of the PV system.

In light of the above, this paper suggests the design of new strategy to detect and diagnose three types of faults frequently occurred in the DC side of PV systems :short circuit faults; open circuit faults and partial shading faults, based on an efficient statistical control charts, EWMA [13], control charts.

The rest of this paper is organized in the following manner: PV module modeling based single diode model topology will be explained briefly in section II, section III will be reserved to the EWMA control chart theory, while the proposed strategy based EWMA control chart will be explained in detail in section VI. Simulation results analysis and discussion will be the aim of section V. finally section VI will be intended to the drawn conclusions.

\section{SINGLE DIODE MODEL:}

From modeling point of view, solar cell/PV module behavior is frequently modeled based on single diode model. This model defines the solar cell/PV module as the following electrical circuit:

while its mathematical formula derived from applying Kirchhoff lows is denoted as:

$$
\mathrm{I}=\mathrm{I}_{\mathrm{ph}}-\mathrm{I}_{0}\left(\exp \left(\frac{\mathrm{q}\left(\mathrm{V}+\mathrm{R}_{\mathrm{s}} \mathrm{I}\right)}{\mathrm{nKT}}\right)-1\right)-\frac{\mathrm{V}+\mathrm{R}_{\mathrm{s}} \mathrm{I}}{\mathrm{R}_{\mathrm{sh}}}
$$

where: I and V denote the solar cell's generated current and voltage respectively; $\mathrm{I}_{\mathrm{ph}}$ : the photo generated currents; $\mathrm{I}_{0}$ : the dark saturation currents; $n$ : the diode's ideality factor; $R_{\mathrm{s}}$ and $\mathrm{R}_{\mathrm{sh}}$ are the series and shunt resistances respectively; $\mathrm{K}$ : Boltzmann constant $\left(1.38064852 \times 10^{-23} \mathrm{~J} \mathrm{~K}^{-1}\right)$,

$\mathrm{T}$ : The cell temperature and $\mathrm{q}$ is the electronic charge $\left(1.60217662 \times 10^{-19} \mathrm{C}\right)$.

As can be observed clearly from (1), single diode model accuracy is heavily based on five unknowns electrical parameters $\left(I_{p h}, I_{0}, n, R_{s}\right.$ and $\left.R_{s h}\right)$, hence the great importance of identifying correctly their corresponding values.

\section{EWMA CONTROL CHART THEORY:}

The exponentially-weighted moving average, EWMA, control chart is one of the most applied statistical method in the field of process monitoring and faults detection [14]. The main idea of using EWMA control chart to detect faults occurrence, consists in examining if the actual measurements (unknown status) are statically different from the non-faulty measurements (a priori known status as nonfaulty process). This control chart have two chief advantages and which are : the high ability to detect small shift in the process mean, since its time-weighted nature, and its great flexibility, which can be seen in the possibility of obtaining high accurate results by adjusting only few parameters. EWMA's has been originally introduced by Roberts et al in [15], then it has been significantly applied in time series analysis [16,17]. Overall several years, EWMA control chart has been applied by lot of engineers and scientists from various areas $[18,19]$.

Assuming that $\left\{\mathrm{x}_{1}, \mathrm{x}_{2}, \ldots, \mathrm{x}_{\mathrm{n}}\right\}$ are the monitored process's collected individual observations, therefore, the statistics of EWMA control chart is computed as follow:

$$
\begin{cases}\mathrm{z}_{\mathrm{t}}=\lambda \mathrm{x}_{\mathrm{t}}+(1-\lambda) \mathrm{z}_{\mathrm{t}-1} & \text { if } \mathrm{t}>0 \\ \mathrm{z}_{0}=\mu_{0} & \text { if } \mathrm{t}=0\end{cases}
$$

where $\mu_{0}$ and $\lambda$ are the mean of the free-fault process's data and the forgetting parameters respectively; $x_{t}$ is actual time observation of the monitored process; $z_{t}$ denotes the monitored statistic (EWMA's output). The value of $\lambda$ (usually $\in[0,1]$ ) defines how fast EWMA forgets the data history; which mean that if $\lambda$ is small, the past observations are more weighted than the actual ones, contrary to the case of large $\lambda$ which leads that the actual observations are more weighted than the past ones. Indeed, small value of the forgetting parameter $\lambda$ is suitable to be used to detect relatively small change in the process mean, while a large value of this parameter should be chosen to detect relatively large change in process mean $[16,19]$.

Using this control chart, the process under monitoring will be declared out of control at time $t$ if its monitored statistic $\mathrm{z}_{\mathrm{t}}$ is outside the control limit boundaries i.e. $\lambda \notin[\mathrm{LCL}, \mathrm{UCL}]$, otherwise, it will be considered under control. where LCL and UCL are EWMA's lower and upper control limits respectively, and they are computed as follow:

$$
\left\{\begin{array}{l}
\mathrm{LCL}=\mu_{0}-\mathrm{L} \sigma_{\mathrm{z}_{\mathrm{t}}} \\
\mathrm{UCL}=\mu_{0}+\mathrm{L} \sigma_{\mathrm{Z}_{\mathrm{t}}}
\end{array}\right.
$$

Where: $\mu_{0}$ is the mean of the free-fault dataset; $\mathrm{L}$ is the control limits' width and which defines the limit of confidence (usually $\mathrm{L}=3$; which corresponds to a false alarm 
rate of $0.27 \%$ ), while $\sigma_{\mathrm{Z}}$ is the standard deviation and it is computed as:

$$
\sigma_{\mathrm{z}_{\mathrm{t}}}=\sigma_{0} \sqrt{\frac{\lambda}{(2-\lambda)}\left(1-(1-\lambda)^{2 \mathrm{t}}\right)}
$$

$\sigma_{0}$ defines the free-fault dataset's standard deviation.

\section{FAUlt DETECTION AND DIAGNOSIS StRATEGY:}

The procedure of using EWMA for fault detection in PV system is summarized as follows:

(a) Compute residuals using fault-free data:

In this step, we compute residuals, which are defined as difference between the measured and predicted data obtained from simulated model.

$$
\operatorname{Res}(\mathrm{X})=\mathrm{X}_{\text {Real }}-\mathrm{X}_{\text {pred }}
$$

where: $\operatorname{Res}(\mathrm{X})$ is the residual of $\mathrm{X}, \mathrm{X}_{\text {Real }}$ and $\mathrm{X}_{\text {Pred }}$ are the real measurement and the prediction values of $X$, respectively. The real measured data are collected from the real system under operation and their predicted values are obtained from a simulated model via a PSIM $^{\mathrm{TM}} / \mathrm{Matlab}^{\mathrm{TM}}$ Co-simulation. Indeed, to simulate the used PV array via this co-simulation, we first identify the five electrical parameters $\left[I_{\mathrm{ph}}, I_{0}, n, R_{s}, R_{s h}\right]$ of a PV module. Then, we construct the simulation model, and compute the residuals of MPP coordinates $\left(\mathrm{I}_{\mathrm{mpp}}, \mathrm{V}_{\mathrm{mpp}}\right.$ and $\left.\mathrm{P}_{\mathrm{mpp}}\right)$. This step 1 can be summarized as follow:

- Identify accurately the five unknown electrical parameters of the employed PV module based on an efficient heuristic optimization technic, Artificial Bee Colony (ABC) algorithm [20-22].

- Simulate the employed PV array under $\mathrm{PSIM}^{\mathrm{TM}} / \mathrm{Matlab}^{\mathrm{TM}}$ co-simulation for a free-faulty (healthy) operating case and store the MPP coordinates of current $I_{m p p}$, Voltage $V_{\text {mpp }}$ and Power $\mathrm{P}_{\mathrm{mpp}}$ as the predicted dataset.

- Compute the residuals of the three stored attributes (Res(Impp); Res(Vmpp); Res(Pmpp) ) based on the real measured and the predicted data.

\section{(b) Compute EWMA control limits:}

In the second step, we compute EWMA control limits for each variable separately (i.e., residuals of DC output current, Res( $\left.\mathrm{I}_{\mathrm{mpp}}\right)$, voltage, $\operatorname{Res}\left(\mathrm{V}_{\mathrm{mpp}}\right)$, and power, $\left.\operatorname{Res}\left(\mathrm{P}_{\mathrm{mpp}}\right)\right)$. The control limits for each are denotes as follows ( $\mathrm{LCL}_{\mathrm{Impp}}$, UCL $\mathrm{L}_{\mathrm{Impp}}$ $\mathrm{LCL}_{\mathrm{Vmpp}}$, UCL $\mathrm{Lmp}_{\mathrm{Vmp}}$, LCL $_{\text {Pmpp }}$, UCL $L_{\text {Pmpp,,) }}$

\section{(c) Compute EWMA statistic for a new data:}

In this phase, testing MPP data of current, voltage and power from the monitored PV system are used to generate residual vectors of current, voltage and power using (5). Then, we compute EWMA monitoring statistics $\left(\mathrm{Z}_{\mathrm{Impp}}, \mathrm{Z}_{\mathrm{Vmpp}}\right.$ and
$\mathrm{Z}_{\mathrm{Pmpp}}$ ) based on the previously computed residual vectors using Equation (2).

(d) Check the fault occurrence and diagnose its nature: When a new sample is available, calculate EWMA based on the ODM model. If EWMA statistics exceeds the confidence limit obtained in the previous step, then a fault is detected. To identify the type of fault we the procedure summarized in Fig.2.

\section{Simulation Results:}

The aim of this part is to assess the efficiency of the proposed method. Toward this end, real meteorological conditions of works (Temperatures $(\mathrm{T})$ and Irradiance $(\mathrm{G})$ ) and MPP coordinates ( $\mathrm{I}_{\mathrm{mpp}}, \mathrm{V}_{\mathrm{mpp}}$, and $\mathrm{P}_{\mathrm{mpp}}$ ) collected from an actual $3.2 \mathrm{KWp} \mathrm{PV}$ plant located within an Algerian Research center (Centre du Développement des Energies Renouvelables (CDER) of Algiers) [23] have been used. This PV plant is composed of two parallel strings of fifteen PV modules type Isofoton $106 \mathrm{~W}-12 \mathrm{~V}$ for each string. The real collected data corresponds to a free-faulty (healthy) system's MPP data of June 24, 2008 day.

As described above, the first step consists in computing the MPP coordinates' residuals (Res $\left(\mathrm{I}_{\mathrm{mpp}}\right)$, Res $\left(\mathrm{V}_{\mathrm{mpp}}\right)$ and $\left.\operatorname{Res}\left(\mathrm{P}_{\mathrm{mpp}}\right)\right)$ which is denoted as the subtraction of the real measure value to the predicted based simulation model value of the corresponding attributes.

In this study, the used simulation model is a PSIM $^{\mathrm{TM}} / \mathrm{Matlab}^{\mathrm{TM}} \mathrm{Co}$-simulation model of the $3.2 \mathrm{KWp} \mathrm{PV}$ plant of CDER. However, to reach the simulation goal, the five unknown electrical parameters $\left(\mathrm{I}_{\mathrm{ph}}, \mathrm{I}_{0}, \mathrm{n}, \mathrm{R}_{\mathrm{s}}\right.$ and $\left.\mathrm{R}_{\mathrm{sh}}\right)$ of its PV module (isofoton 106w-12v) should be accurately identified. To identify unknown electrical parameters of the isofoton 106w-12v PV module, we used a real measured I-V characteristic and an efficient heuristic optimization Algorithm, Artificial Bee Colony (ABC) [20]. The selected parameters are given in table I:

where RMSE describes the root mean square error between the real measured current, and the predicted based $\mathrm{ABC}$ algorithm.

Once the five parameters have been identified, the employed PV plant's model is validated. Validation step is achieved by introducing the identified parameters to the model, then simulate this last under the same meteorological conditions ( $\mathrm{T}$ and $\mathrm{G}$ ) as the measured ones. Finally, the real measured MPP power is compared to the simulated one, as can be appeared in Fig. 3. 


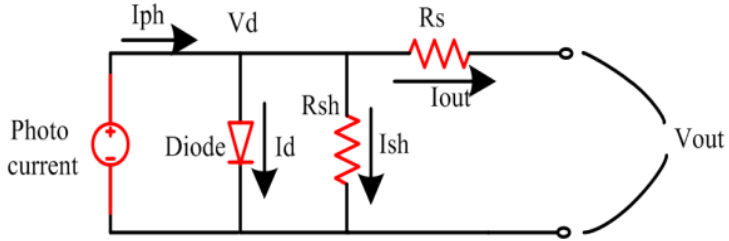

Fig.1. The single diode model of the solar cell/PV module.

TABLE I. Isofoton 106W-12V PV modules' identified parameters

\begin{tabular}{|c|c|c|c|c|c|c|}
\hline Parameters & $\mathbf{I}_{\mathbf{p h}}[\mathbf{A}]$ & $\mathbf{I}_{\mathbf{0}}[\mathbf{A}]$ & $\mathbf{n}$ & $\mathbf{R}_{\mathbf{s}}[\mathbf{\Omega}]$ & $\mathbf{R}_{\text {sh }}[\mathbf{\Omega}]$ & $\mathbf{R M S E}$ \\
\hline Values & 6.54 & $1.11 \mathrm{e}-05$ & 1.66 & 0.147 & 202.6 & 0.015 \\
\hline
\end{tabular}

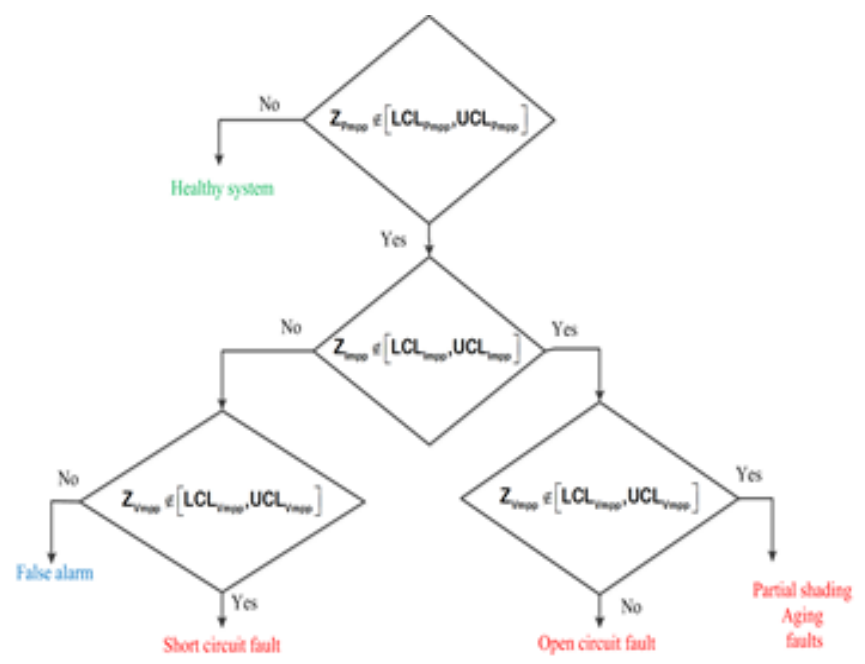

Fig. 2. Fault detection and diagnosis strategy

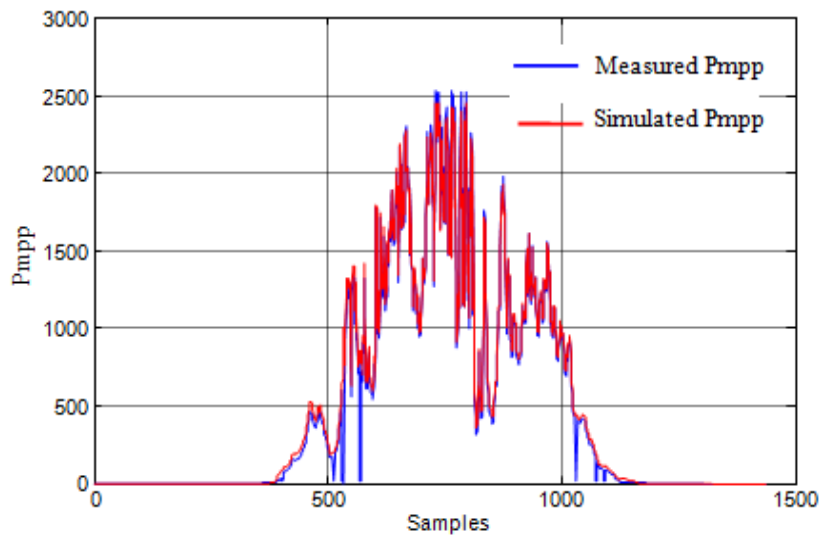

Fig. 3. Real measured versus simulated MPP power.

It can be seen from Fig. 3 that the proposed model performs well in describing the real behavior of the employed PV plant.
After that, the MPP coordinates' residuals of the free-fault PV system are computed based on (5) using the real measured and the predicted data.

Based on these free-fault system's residuals, EWMA's lower and upper control limits, LCL and UCL, for each one of the three attributes ( $\mathrm{I}_{\mathrm{mpp}}, \mathrm{V}_{\mathrm{mpp}}$ and $\mathrm{P}_{\mathrm{mpp}}$ ) are computed using (3).

Free-fault system's monitoring statistics should be also calculated based on (2) to check if they are inside the control limits (LCL and UCL).

Simulation results in terms of free-fault system's MPP residuals computations are depicted in Fig. 4. While, monitoring statistics and theirs corresponding control limits are depicted in Fig. 5.

Based on EWMA control chart in monitoring, we should expect that for the three attributes $\left(\mathrm{I}_{\mathrm{mpp}}, \mathrm{V}_{\mathrm{mpp}}\right.$ and $\left.\mathrm{P}_{\mathrm{mpp}}\right)$, their monitoring statistics data will lie within lower and upper EWMA's control limits.

Results from Fig. 5 show clearly the simulation model's aptitude to describe the free-fault operation case. This conclusion has been directly drawn on the fact that the three monitoring statistics are inside their corresponding EWMA control limits.

Up to now, we have computed the MPP coordinates' residuals and theirs corresponding EWMA's control limits of the free-fault operating PV system.

The goal of the third step is to test the aptitude of our method to detect the DC side faults' occurrence. To this end, three faulty operating cases have been simulated: five modules short-circuited in one string; string completely open-circuited from the array; and finally four modules partially-shaded in the first string operating cases.

The simulated MPP coordinates of the faulty operating cases, will be then used to compute the monitoring statistics for each faulty operating case based on (2).

To test the efficiency of the proposed method, free-fault operation case followed by faulty operation case has been simulated in the following manner:

- Samples from first observation till number 300 correspond to the free-faulty case, while the remaining corresponds to the faulty case (shortcircuit and open circuit faults).

- Samples between observation number 150 and 250 corresponds the faulty operations case (partial shading).

Healthy (free-fault) and faulty systems' monitoring statistics are depicted against free-fault's system EWMA control 
limits for the three faulty operating cases in Fig. 6, Fig. 7 and Fig. 8 respectively.
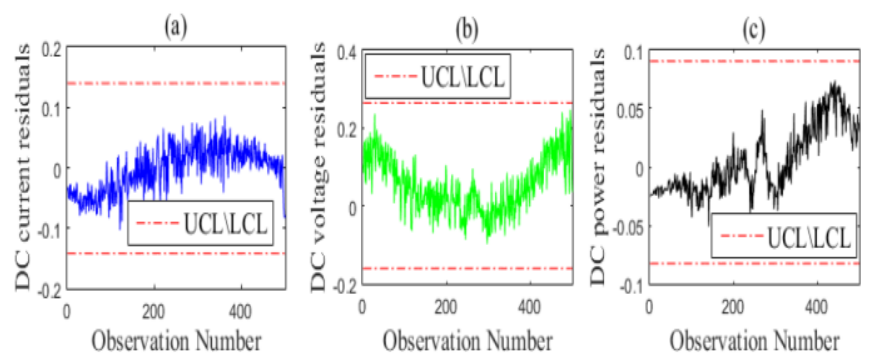

Fig. 4. Free-Fault system's MPP residuals.
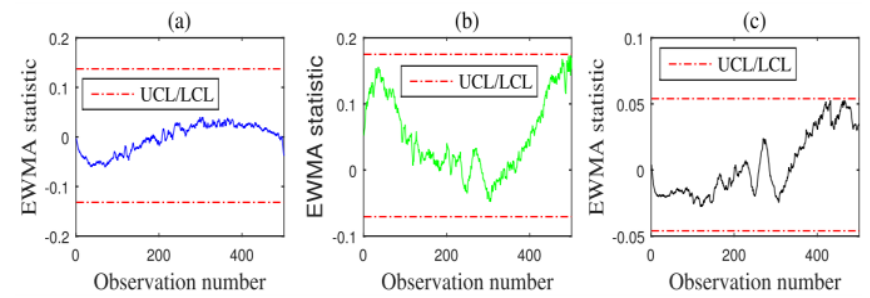

Fig. 5. Monitoring results of EWMA chart for MPP current (a), MPP voltage (b) and MPP power (c) under normal operating conditions.
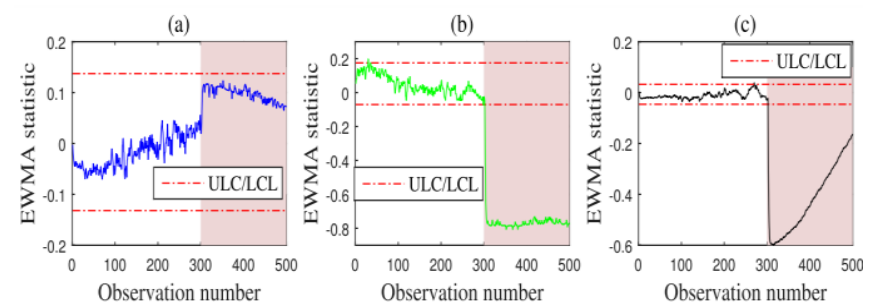

Fig. 6. Monitoring results of EWMA chart for MPP current (a), MPP voltage (b) and MPP power (c) in the presence of five modules shortcircuited fault.
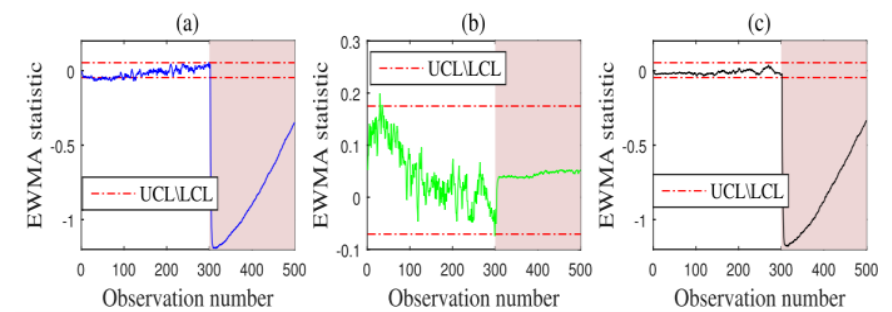

Fig. 7. Monitoring results of EWMA chart for MPP current (a), MPP voltage (b) and MPP power (c) in the presence of string completely opencircuited fault.
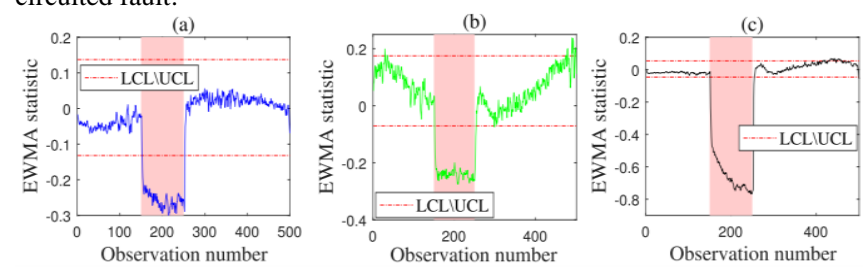

Fig. 8. Monitoring results of EWMA chart for MPP current (a), MPP voltage (b) and MPP power (c) in the presence of four modules partially shaded in the array faulty case.

Finally, faults detections and diagnosis strategy has been proved its high efficiency according to the simulations results.
In fact, since EWMA monitoring statistic of power was inside the free-fault system control limits during its normal operation, then, they move outside these boundaries once a fault has been occurred (Fig. 6(c), Fig. 7 (c), and Fig. 8(c)).

While, faults diagnosis has been achieved correctly based on the explained topology from flowchart depicted in Fig. 2 as follows:

Short circuit fault will be declared if the current monitoring statistics $\left(Z_{\text {Impp }}\right)$ are inside the free-fault system control limits while the voltage ones $\left(\mathrm{Z}_{\mathrm{Vmpp}}\right)$ are outside them (Fig. $6(a ; b))$

Open circuits faults will be triggered if the voltage monitoring statistics $\left(Z_{\mathrm{Vmpp}}\right)$ are inside the free-fault system control limits while the current ones $\left(\mathrm{Z}_{\text {Impp }}\right)$ are outside them (Fig. $7(a ; b))$

While partial shading of modules faults will be declared when both current and voltage monitoring statistics exceed the free-fault system's control limits (Fig. $8(a ; b))$.

Finally, the developed method generates a false alarm when $Z_{\text {Pmpp }}$ are outside the free-fault system's control limits while the two others ( $Z_{\text {Impp }}$ and $Z_{V \mathrm{Vmpp}}$ ) are inside their corresponding free-fault system's EWMA Control limits.

\section{CONCLUSION}

This paper addressed the problem of fault detection and diagnosis in the DC side of a PV system. An innovative statistical model-based fault detection approach has developed to detect and identify faults in a PV system. First, we used a one diode model (ODM) to generate residuals, which are used as the input for the EWMA monitoring scheme. Indeed, DC power residuals are used for faults detection, while DC current and voltage residuals are used for fault identification. This paper demonstrates through practical data from a grid connected PV system at CDER in Algeria, the effectiveness of the ODM-EWMA to detect and identify faults in a PV system, particularly short-circuit faults, open-circuit faults and partial shading faults.

\section{ACKNOWLEDGEMENT}

This publication is based upon work supported by King Abdullah University of Science and Technology (KAUST), Office of Sponsored Research (OSR) under Award No: OSR-2015- CRG4-2582. The authors (Elyes Garoudja and Kamel Kara) thank the SET Laboratory, Department of Electronics, Faculty of Technology, University of Blida 1, Algeria, for continuous support during the study.

\section{REFERENCES}

[1] T. B. Johansson, Renewable energy: sources for fuels and electricity: Island press, 1993. 
[2 ] N. Panwar, S. Kaushik, and S. Kothari, "Role of renewable energy sources in environmental protection: a review," Renewable and Sustainable Energy Reviews, vol. 15, pp. 1513-1524, 2011.

[3] Y. Zhao, R. Ball, J. Mosesian, J.-F. de Palma, and B. Lehman, "Graphbased semi-supervised learning for fault detection and classification in solar photovoltaic arrays," IEEE Transactions on Power Electronics, vol. 30, pp. 2848-2858, 2015.

[4] Y. Zhao, "Fault detection, classification and protection in solar photovoltaic arrays," NORTHEASTERN UNIVERSITY, 2015.

[5] B. Brooks, "The Bakersfield Fire: A Lesson in Ground-Fault Protection," SolarPro Magazine, pp. 62-70, 2011.

[6] "NC PV DG program SEPA presentation," DukeEnergy, pp. 1-14, 2011.

[7] S. Vergura, G. Acciani, V. Amoruso, G. E. Patrono, and F. Vacca, "Descriptive and inferential statistics for supervising and monitoring the operation of PV plants," IEEE Transactions on Industrial Electronics, vol. 56, pp. 4456-4464, 2009.

[8] D. D. Nguyen, B. Lehman, and S. Kamarthi, "Performance evaluation of solar photovoltaic arrays including shadow effects using neural network," in 2009 IEEE Energy Conversion Congress and Exposition, 2009, pp. 3357-3362.

[9] E. Karatepe and T. Hiyama, "Controlling of artificial neural network for fault diagnosis of photovoltaic array," in Intelligent System Application to Power Systems (ISAP), 2011 16th International Conference on, 2011, pp. 1-6.

[10] A. M. Pavan, A. Mellit, D. De Pieri, and S. Kalogirou, "A comparison between BNN and regression polynomial methods for the evaluation of the effect of soiling in large scale photovoltaic plants," Applied energy, vol. 108, pp. 392-401, 2013.

[11] L. Schirone, F. Califano, U. Moschella, and U. Rocca, "Fault finding in a 1 MW photovoltaic plant by reflectometry," in Photovoltaic Energy Conversion, 1994., Conference Record of the Twenty Fourth. IEEE Photovoltaic Specialists Conference-1994, 1994 IEEE First World Conference on, 1994, pp. 846-849.

[12] D. Stellbogen, "Use of PV circuit simulation for fault detection in PV array fields," in Photovoltaic Specialists Conference, 1993., Conference Record of the Twenty Third IEEE, 1993, pp. 1302-1307.

[13] J. Lucas and M. Saccucci, "Exponentially weighted moving average control schemes: properties and enhancements," Technometrics, vol. 32, no. 1, pp. 1-12, 1990.

[14] N. Zerrouki, F. Harrou, Y. Sun, and A. Houacine, "Accelerometer and camera-based strategy for improved human fall detection," Journal of medical systems, vol. 40, no. 12, p. 284, 2016.

[15] Roberts, S.W., "Control chart tests based on geometric moving averages," Technometrics.vol.1, no. 3,p. 239-250, 1959.

[16] Kadri, F., Harrou, F., Chaabane, S., Sun, Y., and Tahon, C., "Seasonal ARMA-based SPC charts for anomaly detection: application to emergency department systems,". Neurocomputing, no. 173, p. 2102 2114, 2016.

[17] Harrou. F, Nounou. M, Nounou. H, and Madakyaru. M, "PLS-based EWMA fault detection strategy for process monitoring," J. Loss Prev. Process Ind. 36:108-119, 2015.

[18] F. Harrou and M. Nounou, "Monitoring linear antenna arrays using an exponentially weighted moving average-based fault detection scheme," Systems Science \& Control Engineering: An Open Access Journal, vol. 2, no. 1, pp. 433-443, 2014.

[19] D. C. Montgomery, "Introduction to statistical quality control," John Wiley\& Sons, New York, 2005.

[20] D. Karaboga and B. Basturk, "On the performance of artificial bee colony (ABC) algorithm," Applied soft computing, vol. 8, pp. 687 697, 2008.

[21] E. Garoudja, K. Kara, A. Chouder, and S. Silvestre, "Parameters extraction of photovoltaic module for long-term prediction using artifical bee colony optimization," in 3rd International Conference on Control, Engineering \& Information Technology (CEIT). IEEE, 2015, pp. 1-6.

[22] S. Abou, A. Chouder, K. Kara, and S. Silvestre, "Artificial bee colony based algorithm for maximum power point tracking (MPPT) for PV systems operating under partial shaded conditions," Applied Soft Computing, vol. 32, pp. 38-48, 2015.
[23] A. Chouder and S. Silvestre, "Automatic supervision and fault detection of PV systems based on power losses analysis," Energy Conversion and Management, vol. 51, no. 10, pp. 1929-1937, 2010. 